\title{
Editorial
}

\section{El efecto reflexivo del espejo sociotécnico}

\author{
Diego S. ACEVEDO A. \\ CIESPAL \\ dacevedo@ciespal.org
}

\begin{abstract}
A partir de los confinamientos producidos por la pandemia del Covid-19 la vida conectiva, que no es un concepto nuevo, tuvo un crecimiento hiper acelerado en todos los rincones del planeta. En este contexto el Centro Internacional de Estudios Superiores en Comunicación para América Latina CIESPAL, junto con reconocidos investigadores de la comunicación que conformaron el grupo editor temático, que se encuentran domiciliados en distintos países, se plantean el reto de investigar Las redes digitales como espejos sociotécnicos de Iberoamérica.
\end{abstract}

Al respecto Rodrigues Costa, Capoano y Barredo (2021) mencionan:

Los espejos sociotécnicos imprimen un reflejo de lo real, prolongan al individuo desde las nuevas posibilidades de colaboración, lo vinculan al colectivo convirtiéndose en aquellas redes esperanzadoras e indignadas, pero también, estas plataformas pueden contribuir al desgaste del tejido social, aislando a los grupos en burbujas de pensamiento, en cámaras de eco que favorecen la polarización y que terminan erosionando a las democracias contemporáneas.

Si bien es cierto que redes colaborativas y tecnología de la comunicación puede ayudar en el progreso de las ciencias, la coyuntura que vive la humanidad ha mostrado que teorías aparentemente sin sentido ni bases científicas, pueden difundirse ampliamente, llegando a ser defendidas enérgicamente por grupos antisistémicos. El origen del problema es más complejo de lo que parece ya que las redes sociales han dado visibilidad a este tipo de información (Nguyen \& Catalan-Matamoros, 2020), que es aceptada por audiencias que sienten el deseo de creer en cosas que, según los estándares intelectuales normales, son inequívocamente contrarios al pensamiento científico (Catalán-Matamoros, 2020). Estudios previos muestran que los intentos explícitos dirigidos a corregir falsas creencias con datos y hechos científicos pueden ser contraproducentes, llevando a las personas a respaldar más firmemente sus creencias previas (Betsch et al., 2013). En otras palabras, los medios digitales actúan como un 
transmisor de desinformación en un entorno donde el conocimiento científico basado en hechos y evidencia no siempre son considerados por el público como la verdad.

A esta realidad se debe sumar la intervención de algoritmos predictivos que detectan el comportamiento de las audiencias (MediaMath, 2018) tales como del Big Data, Machine Learning, Inteligencia Artificial, etc., todos ellos, sistemas enfocados a dirigir el tipo de contenido que consumen las audiencias.

A partir de estas definiciones, el desafío consiste en traer a la palestra del debate trabajos que aporten reflexión crítica sobre el comportamiento y consumo de estas redes y los efectos tanto positivos como negativos en una sociedad hiperinformada.

En este sentido Chasqui, revista latinoamericana de comunicación concentra sus esfuerzos en generar espacios de trabajo colaborativo, libres de influencias hegemónicas, supervisados por la rigurosidad académica y la pertinencia temática, ubicando en la brújula al sur, ese sur pluricultural, multiétnico y colorido, con desafíos locales, hibridismos culturales y nuevas movilidades, como describe Colombo (2001), para quien el siglo XXI se caracterizará, precisamente, por el mestizaje y la importancia emergente de los grupos minoritarios.

\section{Referencias bibliográficas}

Betsch, C., Renkewitz, F., \& Haase, N. (2013). Effect of Narrative Reports about Vaccine Adverse Events and Bias-Awareness Disclaimers on Vaccine Decisions: A Simulation of an Online Patient Social Network. Medical Decision Making, 33(1), 14-25. https://doi. org/10.1177/0272989X12452342

Catalán-Matamoros, D. (2020). La comunicación sobre la pandemia del COVID-19 en la era digital: manipulación informativa, fake news y redes sociales. REVISTA ESPAÑOLA DE COMUNICACIÓN EN SALUD, o, 5-8. doi: http://dx.doi.org/10.20318/recs.2020.5531

Colombo, Sylvia (2001). Literatura: Carlos Fuentes constrói ponte sobre o Atlântico. Folha de S. Paulo. https://www1.folha.uol.com.br/folha/ilustrada/ultgou13889.shtml

MediaMath. (2018). Marketing 2027. Obtenido de http://www.mediamath.com/blog/resource/marketing-2027-survey/

Nguyen, A., \& Catalan-Matamoros, D. (2020). Digital Mis/ Disinformation and Public Engagment with Health and Science Controversies: Fresh Perspectives from Covid-19. Media and Communication, 8(2), 323. https://doi.org/10.17645/mac.v8i2.3352

Rodrigues-Costa, P., Capoano, E., \& Barredo Ibáñez, D. (2021). La vida conectiva. Las redes digitales como espejos sociotécnicos de Iberoamérica. Chasqui. Revista Latinoamericana de Comunicación, 1(147). doi:https://doi.org/10.16921/chasqui.v1i147.4618 